\title{
Cetuximab-loaded Ethylcellulose Polymeric Nanoparticles Decorated with Octreotide (SY)
}

National Cancer Institute

\section{Source}

National Cancer Institute. Cetuximab-loaded Ethylcellulose Polymeric Nanoparticles

Decorated with Octreotide (SY). NCI Thesaurus. Code C158083.

A preparation of ethylcellulose polymeric nanoparticles loaded with cetuximab, a recombinant, chimeric monoclonal antibody directed against the epidermal growth factor (EGFR), and decorated with the somatostatin analog, octreotide, with potential antineoplastic activity. Upon oral administration, the octreotide moiety directs the nanoparticles, which remain inert until a pH of 6.8 is reached, to somatostatin receptors (SSTRs), which are present on the cell membranes of many neuroendocrine tumor (NET) cells. At this $\mathrm{pH}$, cetuximab is selectively released from the ethylcellulose polymer. Cetuximab may then bind to the extracellular domain of EGFR-expressing tumor cells, thereby preventing the activation and subsequent dimerization of the receptor. This may inhibit signal transduction and inhibit tumor cell proliferation in EGFR-dependent tumor cells. EGFR, a member of the EGFR receptor tyrosine kinase family, may be overexpressed on the cell surfaces of various tumor types. 